\title{
In-medium effects on hypernuclear formation
}

\author{
T. Gaitanos ${ }^{\mathrm{a}}$, A.B. Larionov ${ }^{\mathrm{b}, \mathrm{c}}$, H. Lenske ${ }^{\mathrm{a}}$, U. Mosel ${ }^{\mathrm{a}}$, A. Obermann ${ }^{\mathrm{a}}$ \\ ${ }^{a}$ Institut für Theoretische Physik, Universität Giessen, Germany \\ ${ }^{b}$ Frankfurt Inst. for Adv. Studies, J.W. Goethe-Universität, Frankfurt, Germany \\ ${ }^{c}$ Russian Research Center, Kurchatov Institute, Moscow, Russia
}

\begin{abstract}
We study strangeness dynamics in reactions relevant for the formation of multi-strange hypernuclei. In particular, we discuss the in-medium effects on elementary hyperon-nucleon $(Y N)$ channels, which are relevant for the production of hypernuclei at PANDA. The results indicate strong in-medium effects on $Y N$-scattering, which might be important for hypernuclear studies at PANDA.
\end{abstract}

Key words: In-medium hyperon-nucleon interaction, hypernuclei, PANDA experiment

\section{Introduction}

The study of hypernuclei is intimately related to many fundamental aspects of nuclear and hadron physics [1]. Spectroscopy can be at best performed with hyperons, since single hyperons are not Pauli blocked in nucleonic matter and can provide particular clean information on single-particle spectroscopy. The investigation of hypernuclei can provide information also on the still less known hyperon-nucleon and hyperon-hyperon interactions. For the latter the production of double- $\Lambda$ hypernuclei is important, which is one of the main projects of the PANDA Collaboration [2].

Recently, we have studied the formation of $S=-1$ and $S=-2$ hypernuclei in various reactions [3] within a covariant transport model [4]. Here we focus on the discussion of the production mechanisms of double- $\Lambda$ hypernuclei at PANDA. In particular, we examine possible in-medium dependences of hyperon-nucleon interactions important for the formation of hypernuclei in reactions induced by hadrons and heavy-ions.

\section{GiBUU calculations}

The fast non-equilibrium stage of reactions is modelled by the Giessen-Boltzmann-Uehling-Uhlenbeck (GiBUU) transport model [4]. The GiBUU model is based on the following Boltzmann-Uehling-Uhlenbeck equation

$$
\left[k^{* \mu} \partial_{\mu}^{x}+\left(k_{v}^{*} F^{\mu v}+m^{*} \partial_{x}^{\mu} m^{*}\right) \partial_{\mu}^{k^{*}}\right] f\left(x, k^{*}\right)=\mathcal{I}_{\text {coll }} \quad .
$$

Eq. (1) describes the dynamical evolution of the one-body phase-space distribution function $f\left(x, k^{*}\right)$ for the hadrons under the influence of a hadronic mean-field (1.h.s. of Eq. (1)) and binary collisions (r.h.s. of Eq. (1)). Our previous Dirac-Brueckner studies of $N N$ and $Y N$ in-medium interactions [5] are showing that the real parts of the self-energies are well described in Hartree-approximation, at least at low energies. Hence, guided by those results, we obtain a numerically feasible approach by extending the mean-field approximation to dispersive processes with imaginary parts derived by a Monte Carlo procedure (see below). The relativistic mean-field enters into GiBUU through the kinetic 4-momenta $k^{* \mu}=k^{\mu}-\Sigma^{\mu}$ and the effective (Dirac) masses $m^{*}=M-\Sigma_{s}$, with the vector and scalar self-energy components given by $\Sigma^{\mu}=g_{\omega} \omega^{\mu}+\tau_{3} g_{\rho} \rho_{3}^{\mu}, \Sigma_{s}=g_{\sigma} \sigma$, with the isoscalar, scalar $\sigma$, the isoscalar, vector $\omega^{\mu}$ meson fields and the third isospin-component of the isovector, vector $\rho_{3}^{\mu}$ meson field. The meson-nucleon coupling constants $g_{i}(i=\sigma, \omega, \rho)$ and also the additional parameters of the non-linear self-interactions of the $\sigma$ meson (not shown here) are taken from the widely used NL parametrizations, see [3] for details. The antinucleon-meson coupling constants are rescaled by a phenomenological constant such, to reproduce data in high-energy antiproton-induced reactions [3].

Furthermore, the collision term is treated in a parallel ensemble algorithm incorporating the standard parametrizations for the cross sections of various binary processes [3, 4]. For the present calculations we follow the usual scheme Preprint submitted to Nuclear Physics A

September 21, 2018 

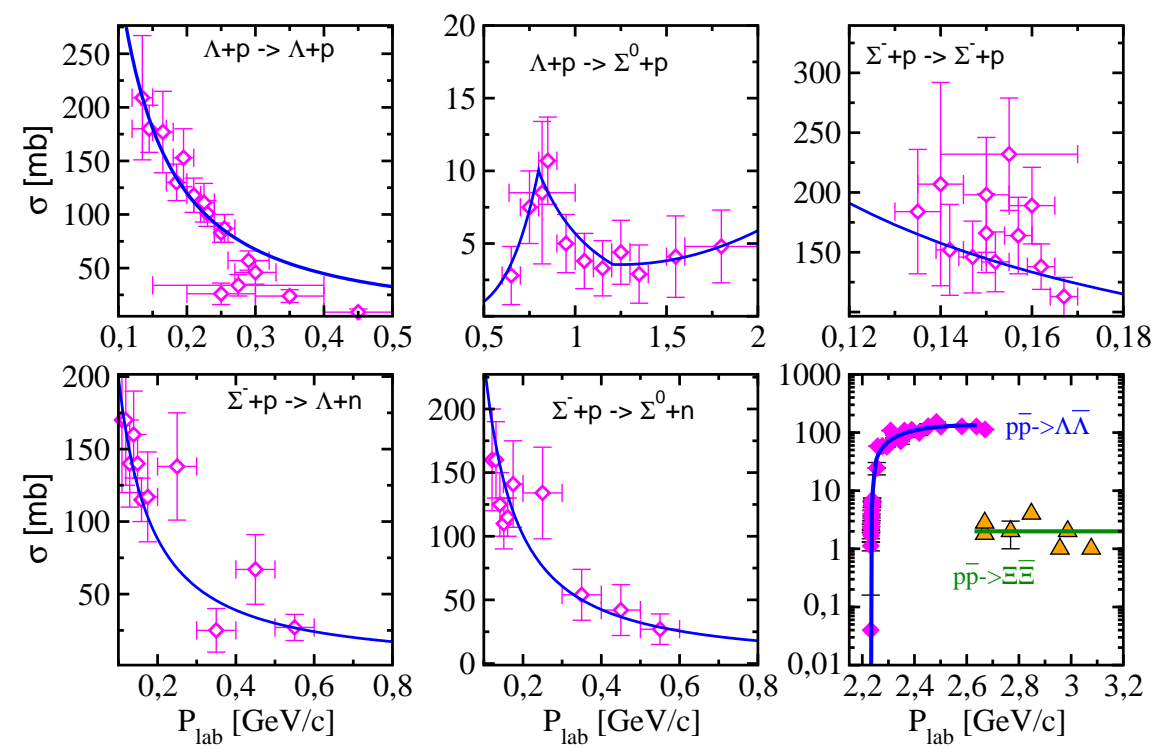

Figure 1: Cross sections as function of beam momentum for various channels (as indicated) relevant for the production of hypernuclei. Theoretical parametrizations (solid curves) are compared with data (symbols, taken from [6]).

of using empirical cross section data. The approach discussed in the next section will allow us to overcome that limitation in the near future.

GiBUU results on fragment formation and on multi-strange $\Lambda$-hypernuclei at PANDA have been published in Refs. [3]. Except of a proper description of fragmentation [3], the theoretical description of the various elementary channels for strangeness production is also important. Fig. 11 shows some examples for secondary and primary hyperon production channels, where one realizes the need of more precise measurements of such elementary input. Note that not for all the relevant channels data exist. In fact, the channel $\Xi N \rightarrow \Lambda \Lambda$, which is crucial for the formation of double- $\Lambda$ hypernuclei at PANDA, completely relies on theoretical estimates [3].

Fig. [2] shows GiBUU results for the time evolution of strangeness with $S=-1$ and $S=-2$, for $\Xi$-induced reactions at three $\Xi$-energies. The production of bound $\Lambda$ particles strongly decreases with $\Xi$-energy. This is due to the enhanced repulsive character of the mean-field at large positive energies, as typical for baryon mean-field selfenergies, but also due to the strong decrease of the elementary $\Xi N \rightarrow \Lambda \Lambda$ cross section with rising energy. These results are compatible with our previous studies [3].

\section{In-medium hyperon-nucleon calculations}

Reactions induced by (anti)baryons and heavy-ions take place not in free space, but inside a dense hadronic environment. Thus, the in-medium dependence of the same elementary channels is indispensable for the production of hypernuclei, apart from the mean-field effects. First studies on this direction have been already started within the Bethe-Salpeter formalism for $Y N$ in-medium scattering. This is done by solving the Lippmann-Schwinger (LS) equation for the R-matrix, given in short-hand notation by

$$
R=V+\mathcal{P} \int V G Q R
$$

where $V$ is the underlying $Y N$-potential in free space, $G$ the propagator for intermediate states and $Q$ the Pauli operator for intermediate states. The integration is performed over intermediate states and $\mathcal{P}$ denotes the principal value. Since we account for the coupling of the various $Y N$ channels of same strangeness $S$ and total charge, the LS equation, in fact, has a matrix structure. The free space $V_{Y N}$ potential is obtained by rescaling the $N N$ coupling constants of the Bonn potential by fits to the scarce data quoted above and results by the Juelich group [7, 8]. The parameters 


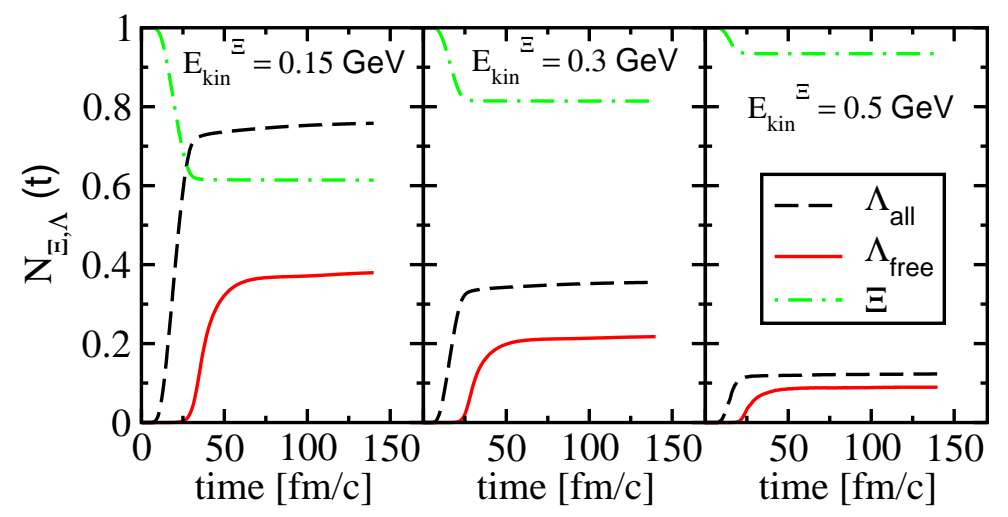

Figure 2: GiBUU results for the time dependence of all and free $\Lambda$ 's and of $\Xi$ 's for central $\Xi$-induced reactions on ${ }^{64} \mathrm{Cu}$-target at beam energies as indicated.

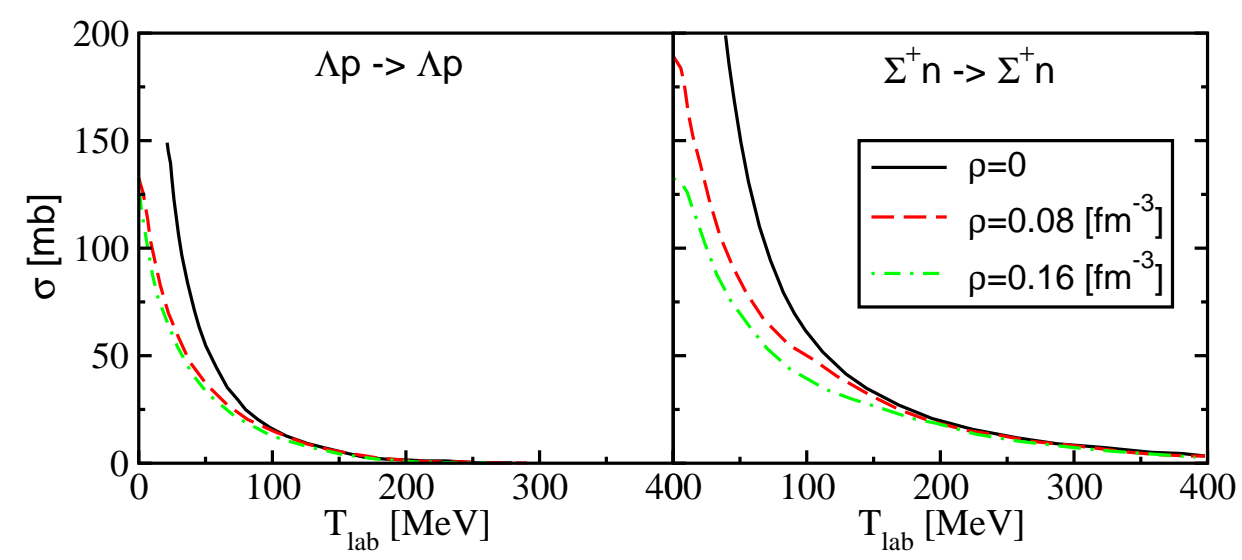

Figure 3: Energy dependence of elastic in-medium cross sections for $\Lambda N$ and $\Sigma N$ scattering (as indicated) at densities of $\rho=0,0.08,0.16\left[\mathrm{fm}^{-3}\right.$ ] (solid, dashed and dot-dashed, respectively) [7].

are determined such that one first starts at low energies where $\Lambda \Sigma$ coupling does not occur, and obtains the rescaling parameter $b_{\Lambda N}$ for the simple case of $\Lambda N \rightarrow \Lambda N$. The remaining parameters are determined at higher energies above the kinematical thresholds of the channels $\Sigma N \rightarrow \Sigma N$ and $\Lambda N \rightarrow \Sigma N$. This results in a reduction of the NN coupling parameters by factors $b_{\Lambda N, \Sigma N} \simeq 0.8-0.86$ for $\Lambda N \rightarrow \Lambda N$ and $\Sigma N \rightarrow \Sigma N$, and $b_{\Lambda \Sigma}=0.02$ for $\Lambda N \rightarrow \Sigma N$. Finally, in-medium effects are incorporated by solving the LS equation with the inclusion of Pauli projector $Q$. Pauli-blocking is in fact the leading order source of medium effects. Since the self-energies are contributing only from second order on, they have been left out at this moment.

Fig. 3 shows the energy dependence of $Y N$ elastic in-medium cross sections at different densities. Inside nuclear matter, the $Y N$ interaction strength is further reduced, mainly by Pauli-blocking of the intermediate nucleon states. As seen from Fig. 3, the reduction increases with density, leading at saturation density to a suppression of the cross sections by about $50 \%$. Our results indicate that one must expect important in-medium effects of $Y N$ secondary scattering in reactions induced by heavy-ions (HypHI experiment [9]) and by antiprotons (PANDA experiment [2]) and, therefore, on the production of hypernuclei. For a proper treatment in transport-theoretical calculations these inmedium dependences have to be implemented in transport codes, by parametrizing the density and energy dependence of the in-medium cross sections. This is feasible and presently in progress.

A suitable measure for the interaction strength at threshold is obtained from the low-energy effective range expansion of the $l=0$ phase shift

$$
\frac{k}{\tan \delta}=-\frac{1}{a_{s}}+\frac{1}{2} k^{2} r_{s}
$$




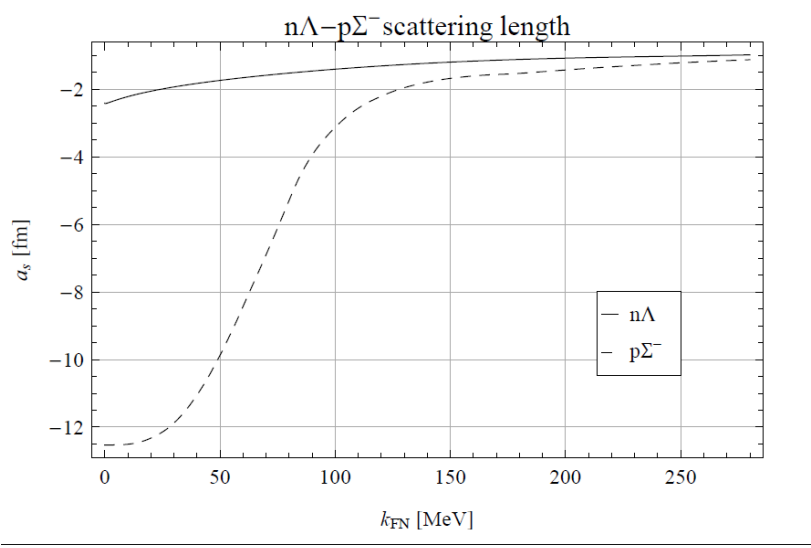

Figure 4: Scattering length $a_{s}$ for the channels as indicated as function of Fermi-momentum of the nucleonic medium.

leading to the effective range $r_{s}$ and to the scattering length $a_{s}$, which is proportional to the volume integral of the underlying $Y N$ potential. In Fig. 4, $a_{s}$ is displayed for the $n \Lambda$ and $p \Sigma^{-}$systems as function of $k=k_{F}$, i.e., the onshell momentum is taken at the Fermi-momentum of the nucleonic background medium. Close to saturation a strong reduction, especially in the $p \Sigma^{-}$-channel, is observed, approaching the strength of the $n \Lambda$-channel.

\section{Final remarks}

In summary, the production of hypernuclei in reactions induced by heavy-ions and (anti)protons opens the opportunity to explore the less known hyperon-nucleon and hyperon-hyperon interactions at densities close to saturation and beyond. First transport-theoretical studies within the GiBUU transport model have been already performed. Here the in-medium effects of various channels relevant for the production of hypernuclei have been studied and have been found to be important, in particular, at low energies. Since the hypernuclear formation takes place inside a rather highly excited hadronic environment, possible in-medium effects, which emerge at finite baryon density, have to be taken into account in transport simulations of reactions.

\section{Acknowledgments}

This work is supported by BMBF contract GILENS 06, DFG contract Le439/9-1 and HIC for FAIR.

\section{References}

[1] E. Friedmann, A. Gal, Phys. Rep. 452 (2007) 89.

[2] A. S. Lorente, et al., Phys. Lett. B697 (2011) 222.

[3] T. Gaitanos, et al., Nucl. Phys. A881 (2012) 240, and references therein.

[4] O. Buss, et al., Phys. Rep. 512 (2012) 1.

[5] F. de Jong, H. Lenske, Phys. Rev. C58 (1998) 890; C. M. Keil, F. Hofmann, H. Lenske, Phys. Rev. C61 (2000) 064309.

[6] The Landolt-Börnstein database, vol. 12b, DOI: 10.1007/b35211.

[7] A. Obermann, Master's Thesis, 2011, Universität Giessen, unpublished, and references therein; J. Haidenbauer, private communication, 2011.

[8] A. Reuber, et al., Nucl. Rhys. A608 (1996) 243.

[9] T. R. Saito, et al., Nucl. Phys. A835 (2010) 110. 